\title{
Sliding mode control of a differential-drive mobile robot following a path*
}

\author{
Arnau Dòria-Cerezo ${ }^{1}$, Domingo Biel $^{2}$, Josep M. Olm³ and Víctor Repecho ${ }^{4}$
}

\begin{abstract}
This paper presents a control algorithm for a differential-drive robot following a path. The main contributions are: a new control formulation that does not require the robot global position, and a nonlinear controller based on the sliding mode control approach that guarantees stability in both forward and backward motion. Numerical simulations are provided to validate the proposed algorithm.
\end{abstract}

\section{INTRODUCTION}

Differential-drive robots (also known as unicycle twowheeled robot) is attracting the interest of many researchers, from young students (thanks to its mechanical simplicity) to more complex applications such as the Micro Robot Soccer Tournament [1], cooperative control formation [2] [3] [4], or platooning [5], among others.

The control of differential-drive robots usually follows two approaches: positioning (or point-to-point motion), and path (or trajectory) following [6]. The main issue when positioning the robot comes from the fact that the vehicle has a first-order nonholonomic constraint (also called the lateral zero-speed constraint), in such a way that its linear velocity is always aligned with the longitudinal axis, see Figure 1 [6]. Several papers have considered to this problem, as for example [7].

The path following problem consists in tracking a desired path defined by the plane coordinates, $\rho(x, y)$ [6]. Several control strategies have been used to tackle the path following problem, such as backsteeping [8], feedback linearisation [6], fuzzy logic controllers [1], Lyapunov methods [9], adaptive controllers [10], and sliding mode control [12] [13] [11], among others. The main drawback of tracking a trajectory defined by the position in the plane $x, y$ is that it requires knowledge of the global position. Alternatively, and considerably less used, the path tracking problem can be formulated in terms of regulating to zero the distance to the path modeling in a Frénet Frame, see [14] or [15]. The main

\footnotetext{
*The work of A. Dòria-Cerezo, D. Biel, and J.M. Olm was partially supported by the Government of Spain through the Agencia Estatal de Investigación Project DPI2017-85404-P and by the Generalitat de Catalunya through the AGAUR Project 2017 SGR 872.

${ }^{1}$ Arnau Dòria-Cerezo is with the Dept. of Electrical Engineering and Inst. of Industrial and Control Engineering, Universitat Politècnica de Catalunya, Barcelona, Spain arnau. doria@upc.edu

${ }^{2}$ Domingo Biel is with the Dept. of Electronic Engineering and Inst. of Industrial and Control Engineering, Universitat Politècnica de Catalunya, Barcelona, Spain domingo.biel cupc. edu

${ }^{3}$ Josep M. Olm is with the Dept. of Mathematics and Inst. of Industrial and Control Engineering, Universitat Politècnica de Catalunya, Barcelona, Spain josep.olmaupc.edu

${ }^{4}$ Víctor Repecho is with the Inst. of Industrial and Control Engineering, Universitat Politècnica de Catalunya, Barcelona, Spain victor.repecho.deldupc.edu
}

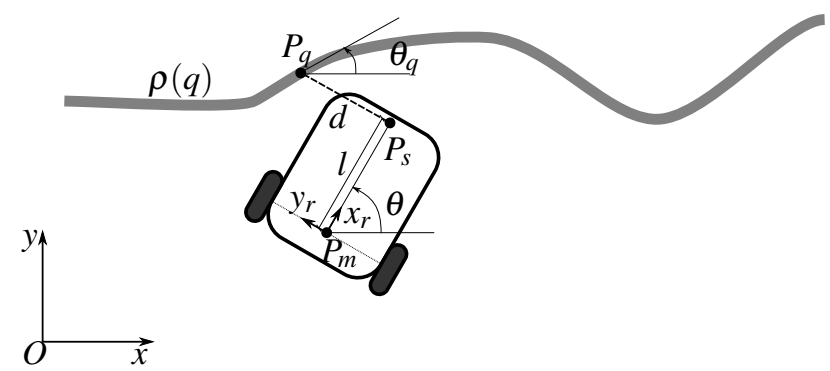

Fig. 1. A two-wheel differential drive mobile robot tracking a path.

advantage is that the MIMO control problem is reduced to a SISO one. In the mentioned papers the distance to the path is obtained projecting the point $P_{s}$ orthogonally on $\rho(x, y)$, see Figure 1, which means that can not be directly measurable form onboard sensors.

In this paper, the path following problem is stated by using the distance orthogonal to the longitudinal axis of the robot. Then, this distance can be obtained from on-board sensors, as for example line optical or image-based sensors, and global position measures are not required. This formulation implies a change of coordinates that translates the global position to distance to the path and, consequently, more complex and nonlinear dynamics arise. On the other hand, the problem of following a path backwards has been disregarded in the literature. To the best of the authors' knowledge, only [9] considers negative longitudinal speeds.

The main contribution of this paper is the design of a control algorithm for a differential-drive robot following a path, that allows both forward and backward motion. The paper is organised as follows: in Section II, the robot model and control problem formulation are presented. The control design, based on sliding mode control, and a stability analysis, are presented in Section III. Section IV includes the observer design for the deviation angle between the robot and the desired path, and Section $\mathrm{V}$ presents simulation results. Finally, Conclusions are drawn in Section VI.

\section{MODEL OF A DIFFERENTIAL-DRIVE ROBOT FOLLOWING A PATH}

The control problem consists in a two-wheel mobile robot tracking a path with a certain longitudinal speed, $v_{x}$. In this paper we consider that an optical sensor, placed at point $P_{S}$, is able to measure the orthogonal distance to the robot axis $\overline{P_{m} P_{s}}$, see Figure 1. The goal is to place (i.e. to regulate) point $P_{s}$ on the curve parametrised as $\rho(q)$, where $\rho(t) \in$ 
$\mathbb{R}^{2}$. Therefore, distance between points $P_{s}$ and $P_{q}$, namely $d=\operatorname{dist}\left(P_{s}, P_{q}\right)$, where $P_{q}$ is the intersection of the desired path $\rho(q)$ with a straight line on the direction $y_{r}$ that passes through $P_{s}$, is aimed to be $d=0$.

The kinematic model for the unicycle-type mobile robot with respect to point $P_{m}\left(x_{m}, y_{m}\right)$ (located in the middle of the wheels axis) is given by ${ }^{1}$

$$
\begin{aligned}
\dot{x}_{m} & =v \cos (\theta) \\
\dot{y}_{m} & =v \sin (\theta) \\
\dot{\theta} & =-u
\end{aligned}
$$

with $v=\frac{r}{2}\left(\omega_{l}+\omega_{r}\right), u=\frac{r}{2 R}\left(\omega_{l}-\omega_{r}\right)$ where $x, y$, are the global reference coordinates for $P_{m}, \theta$ is the orientation of the mobile robot, $r$ is the wheels' radius, $R$ is the distance between the two actuated wheels, and $\omega_{r}\left(\omega_{l}\right)$ is the angular velocity of the right (left) rear wheel.

The control problem is defined as: given a desired longitudinal speed, $v_{x}$, the point $P_{S}$ is to be placed on the path $\rho(q)$. The dynamics is obtained similarly to the Frénet frame-based model presented in [14], where point $P_{q}\left(\rho_{x}(q), \rho_{y}(q)\right)$ is defined from the tangent distance between the path $\rho(q)$ and point $P_{s}$. From Figure 1 one can see that $\overrightarrow{O P_{q}}=\overrightarrow{O P_{m}}+\overrightarrow{P_{m} P_{q}}$, or

$$
\left(\begin{array}{l}
\rho_{x}(q) \\
\rho_{y}(q)
\end{array}\right)=\left(\begin{array}{l}
x_{m} \\
y_{m}
\end{array}\right)+R(\theta)\left(\begin{array}{l}
l \\
d
\end{array}\right)
$$

where

$$
R(\theta)=\left(\begin{array}{cc}
\cos \theta & -\sin \theta \\
\sin \theta & \cos \theta
\end{array}\right) .
$$

Differentiating (4) and using (1)-(3), is possible to determine the motion equations in terms of $d, q$ and $\theta$ :

$$
\begin{aligned}
\dot{d} & =l u-(v+d u) \tan \left(\theta-\theta_{q}\right) \\
\dot{q} & =\frac{1}{\cos \left(\theta-\theta_{q}\right)}(v+d u) \\
\dot{\theta} & =-u
\end{aligned}
$$

where $\frac{\partial \rho_{x}}{\partial q}=\cos \theta_{q}$ and $\frac{\partial \rho_{y}}{\partial q}=\sin \theta_{q}$ have been used. Defining a deviation angle $\theta_{e}=\theta-\theta_{q}$, the dynamics boils down to

$$
\begin{aligned}
\dot{d} & =l u-(v+d u) \tan \left(\theta_{e}\right) \\
\dot{q} & =\frac{1}{\cos \theta_{e}}(v+d u) \\
\dot{\theta}_{e} & =-u-\frac{c(q)}{\cos \theta_{e}}(v+d u)
\end{aligned}
$$

where $c(q)=\frac{\partial \theta_{q}}{\partial q}$ is the curvature ${ }^{2}$ of $\rho(q)$. Let us assume that $v$ is designed such that $\dot{q}=v_{x}$ is guaranteed. Then, the remaining dynamics from (9)-(11) yields

$$
\begin{aligned}
\dot{d} & =l u-v_{x} \sin \left(\theta_{e}\right) \\
\dot{\theta}_{e} & =-u-c(q) v_{x},
\end{aligned}
$$

where the curvature is treated as a disturbance.

\footnotetext{
${ }^{1}$ In many references, $u$ is defined such that positive values increase $\theta$, but here we take (3) to keep a positive relationship with respect to $d$.

${ }^{2}$ For simplicity, in the sequel the argument of $c(q)$ is omitted.
}

Remark 1: Consider a constant curvature $c=$ ct and a desired working point given by any $d^{\text {ref }}=0$. Then, from (12) and (13), the required control value, $u^{*}$, that ensures $\dot{d}=\dot{\theta}_{e}=0$ is

$$
u^{*}=-c v_{x},
$$

and the corresponding deviation angle is

$$
\theta_{e}^{*}=\arcsin (-c l)
$$

which yields the maximum curvature constraint, given by

$$
c<c_{\text {MAX }}=\frac{1}{l} .
$$

Along this paper we will assume a constant curvature $c=\mathrm{ct}$.

\section{Sliding Mode Control Design}

In this Section the Sliding Mode Control technique is applied to the two-wheel mobile robot. The control goal is to achieve a perfect track to a given path defined as $d^{\text {ref }}=0$.

\section{A. First order Sliding Mode Controller}

According to the control objective, we define the switching function as

$$
\sigma=d+\beta \tilde{\theta}_{e}
$$

where $\tilde{\theta}_{e}=\theta_{e}-\theta_{e}^{*}$ and $\beta$ is a gain to be defined. From (12), the equivalent control, defined so that $\dot{\sigma}=0$, is

$$
u_{e q}=\frac{v_{x}}{l-\beta}\left(\sin \theta_{e}+\beta c\right)
$$

and the ideal sliding dynamics (the remaining dynamics when $\sigma=0$ and $u=u_{e q}$ ) can be obtained replacing (18) in (13) and using (17)

$$
\dot{\theta}_{e}=-\frac{v_{x}}{l-\beta}\left(\sin \theta_{e}+c l\right) .
$$

Proposition 1: Consider the nonlinear dynamical system (19). The equilibrium point of (19) given by $\theta_{e}^{*}=$ $\arcsin (-c l)$ is locally asymptotically stable if $\frac{v_{x}}{l-\beta}>0$, with the domain of attraction given by $\theta_{e} \in\left(-\pi-\theta_{e}^{*}, \pi-\theta_{e}^{*}\right)$.

Proof: The phase portrait $\theta_{e}, \dot{\theta}_{e}$ of (19) for $\frac{v_{x}}{l-\beta}>0$ is shown in Figure 2. The arrows in the figure denote the direction of motion, and for any point in $\left(-\pi-\theta_{e}^{*}, \pi-\theta_{e}^{*}\right)$ the motion goes to $\theta_{e}^{*}$. It is seen from the phase portrait that the equilibrium point $\theta_{e}^{*}$ is stable.

Finally, differentiating the switching function (17),

$$
\dot{\sigma}=(l-\beta) u-v_{x}\left(\sin \left(\theta_{e}\right)+\beta c\right),
$$

it follows immediately that the control action

$$
u=u_{e q}-\frac{k}{l-\beta} \operatorname{sign}(\sigma)
$$

where $k>0$, ensures the sliding condition $\sigma \dot{\sigma}<0$, and guarantees that $\sigma=0$ is reached in finite time.

Alternatively, considering a switching control action $u \in$ $\left(u^{\min }, u^{\max }\right)$, and assuming that the equivalent control lies in the interval $\left(u^{\min }, u^{\max }\right)$, the switching policy becomes

$$
u=\left\{\begin{array}{lll}
u^{\min } & \text { if } & \sigma>0 \\
u^{\max } & \text { if } & \sigma<0
\end{array} .\right.
$$




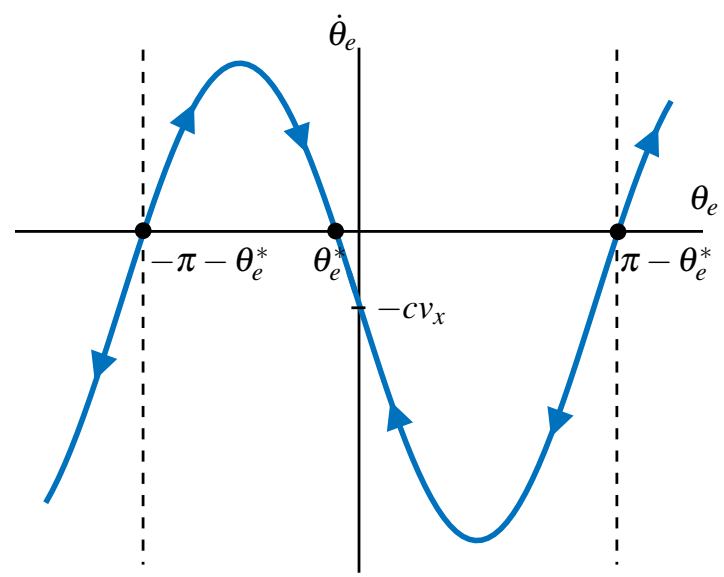

Fig. 2. Phase portrait of the first order system (19).

Remark 2: The stability condition in Proposition 1 suggests that, when the robot moves forward (i.e. $v_{x}, l>0$ ), the $\beta$ gain can be set to zero $(\beta=0)$ and the switching function (17) simplifies to

$$
\sigma=d .
$$

\section{B. Including an integral action}

The control law proposed in (21) requires knowledge of $\theta_{e}^{*}$, which depends on the curvature, $c$, see (15). In a practical scenario, $c$ will be unknown. A way to ensure that $d=0$ when $\sigma=0$, is adding an integral action in the switching function as

$$
\sigma=d+\beta \theta_{e}+z
$$

where

$$
\dot{z}=k_{i} d \text {. }
$$

The equivalent control and the ideal sliding dynamics are,

$$
u_{e q}=\frac{1}{l-\beta}\left(v_{x}\left(\sin \theta_{e}+\beta c\right)-k_{i} d\right)
$$

and

$$
\begin{aligned}
\dot{\theta}_{e} & =-\frac{1}{l-\beta}\left(v_{x} \sin \theta_{e}+k_{i} \beta \theta_{e}\right)-\frac{k_{i}}{l-\beta} z-\frac{l}{l-\beta} v_{x} c \\
\dot{z} & =-k_{i} \beta \theta_{e}-k_{i} z,
\end{aligned}
$$

respectively.

The ideal sliding dynamics defined by (27)-(28) is locally analysed. Its linear approximation in a neighbourhood of the equilibrium point $\left(\theta_{e}^{*},-\beta \theta_{e}^{*}\right)$ results in the state matrix

$$
A=\left(\begin{array}{cc}
-\frac{v_{x} \cos \theta_{e}^{*}+k_{i} \beta}{l-\beta} & -\frac{k_{i}}{l-\beta} \\
-k_{i} \beta & -k_{i}
\end{array}\right)
$$

the characteristic polynomial being

$$
D(s)=s^{2}+\frac{v_{x} \cos \theta_{e}^{*}+k_{i} \beta}{l-\beta} s+\frac{v_{x} k_{i} \cos \theta_{e}^{*}}{l-\beta} .
$$

Then, asymptotic stability of the equilibrium is guaranteed whenever

$$
\begin{aligned}
\frac{v_{x} \cos \theta_{e}^{*}+k_{i} \beta}{l-\beta} & >0 \\
\frac{v_{x} k_{i} \cos \theta_{e}^{*}}{l-\beta} & >0 .
\end{aligned}
$$

Additionally, notice that the control parameters $\beta, k_{i}$ can be used to assign the two poles of the ideal sliding dynamics (presuming $\theta_{e}^{*}$ known). Finally, the control action is defined by (21) with (24) and (26).

\section{OBSERVER DESIGN}

The controllers proposed in Section III require the knowledge of $\theta_{e}$. However, this signal is unavailable in a real plant. In this section, the system is linearised, the observability property of the system is verified and then, a Luenberger observer for $\theta_{e}$ is proposed.

The linear approximation of (12)-(13) with respect to the point defined by $d^{*}=0$ is given by

$$
\left(\begin{array}{c}
\dot{d} \\
\dot{\tilde{\theta}}_{e}
\end{array}\right)=\left(\begin{array}{cc}
0 & -v_{x} \cos \theta_{e}^{*} \\
0 & 0
\end{array}\right)\left(\begin{array}{c}
d \\
\tilde{\theta}_{e}
\end{array}\right)+\left(\begin{array}{c}
l \\
-1
\end{array}\right) \tilde{u}
$$

where $\tilde{\theta}_{e}=\theta_{e}-\theta_{e}^{*}$ and $\tilde{u}=u-u^{*}$.

The observability matrix of the system defined in (33) with output $y=d$ is

$$
W_{o}=\left(\begin{array}{cc}
1 & 0 \\
0 & -v_{x} \cos \theta_{e}^{*}
\end{array}\right)
$$

which is full rank since $v_{x} \neq 0$. Consequently, the system is observable whenever is moving.

The proposed Luenberger observer is

$$
\left(\begin{array}{c}
\dot{\hat{d}} \\
\dot{\hat{\theta}}_{e}
\end{array}\right)=\left(\begin{array}{cc}
0 & -v_{x} \cos \theta_{e}^{*} \\
0 & 0
\end{array}\right)\left(\begin{array}{c}
\hat{d} \\
\hat{\theta}_{e}
\end{array}\right)+\left(\begin{array}{c}
l \\
-1
\end{array}\right) \tilde{u}+\left(\begin{array}{c}
L_{1} \\
L_{2}
\end{array}\right)(d-\hat{d}),
$$

where the state matrix of the observer is

$$
A_{o}=\left(\begin{array}{cc}
-L_{1} & -v_{x} \cos \theta_{e}^{*} \\
-L_{2} & 0
\end{array}\right)
$$

the characteristic polynomial being

$$
D_{o}(s)=s^{2}+L_{1} s+L_{2} v_{x} \cos \theta_{e}^{*} .
$$

The asymptotic stability of $\left(0, \theta_{e}^{*}\right)$ is guaranteed with $L_{1}>0$ and $-L_{2} v_{x} \cos \theta_{e}^{*}>0$.

\section{Simulation Results}

The differential-drive robot with the control algorithm proposed in (21) has been tested in numerical simulations. The nonlinear model has been implemented in Matlab/Simulink for three different paths: i) two straight lines with an angle of $30^{\circ}$, ii) a half circle, and iii) a sinusoidal path. This corresponds to different curvatures: $c=0, c=\mathrm{ct}$, and $c \neq \mathrm{ct}$, respectively. The robot parameters are $l=0.05 \mathrm{~m}, R=0.05 \mathrm{~m}$ and $r=0.02 \mathrm{~m}$, with a desired longitudinal speed of $v_{x}=$ $0.15 \mathrm{~m} / \mathrm{s}$ (when moving forward) and $v_{x}=-0.15 \mathrm{~m} / \mathrm{s}$ (when moving backwards).

The observer and control parameters have been set to $L_{1}=$ 500 and $L_{2}=-5 \cdot 10^{4} \operatorname{sign}\left(v_{x}\right), k=0.1, \beta=2 l\left(1-\operatorname{sign}\left(v_{x}\right)\right)$, $k_{i}=0.25$, and the sign function has been approximated by a hysteresis with thresholds $\varepsilon= \pm 10^{-3}$.

The first test consisted in a path of two straight lines with an angle of with $30^{\circ}$ (curvature $c=0$ ). The initial 

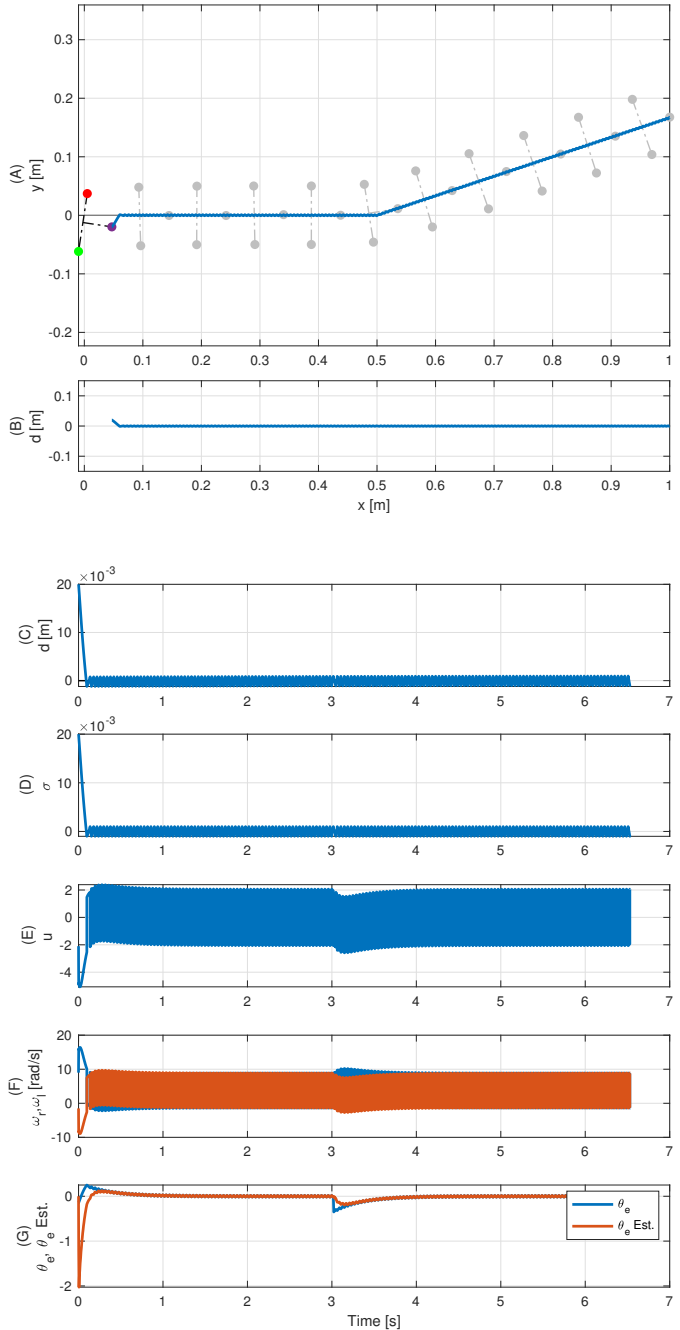

Fig. 3. Simulation results for two straight lines with an angle of $30^{\circ}$, moving forwards $\left(v_{x}>0\right)$ : (A) $x, y$ plane plot: distance sensor (blue dot), left wheel (red dot) and right wheel (green dot); (B) distance to the path, $d$, with respect to $x$; (C) distance to the path, $d$ (in blue), and its reference, $d^{\text {ref }}=0$ (in black); (D) sliding variable, $\sigma$; (E) control action, $u$; (F) wheel's speeds, $\omega_{l}, \omega_{r}$; and $(\mathrm{G})$ estimated deviation angle $\hat{\theta}_{e}$ (in red), with respect to the real one $\theta_{e}$ (in blue).

conditions were $x_{r}(0)=-0.0024 \mathrm{~m}, y_{r}(0)=-0.0123 \mathrm{~m}$ and $\theta(0)=0.15 \mathrm{rad}$, which corresponds to $d(0)=0.025 \mathrm{~m}$, when moving forwards, and $x_{r}(0)=0.9036 \mathrm{~m}, y_{r}(0)=0.1228 \mathrm{~m}$ and $\theta(0)=0.15 \mathrm{rad}$, which corresponds to $d(0)=0.02 \mathrm{~m}$, when moving backwards.

The second test consisted in a circular path with radius $0.5 \mathrm{~m}$ (constant curvature, $c=2$ ). The initial conditions were $x_{r}(0)=-0.0024 \mathrm{~m}, y_{r}(0)=-0.0123 \mathrm{~m}$ and $\theta(0)=0.15 \mathrm{rad}$, which corresponds to $d(0)=0.025 \mathrm{~m}$ when moving forwards, and $x_{r}(0)=0.9036 \mathrm{~m}, y_{r}(0)=0.1228 \mathrm{~m}$ and $\theta(0)=0.15 \mathrm{rad}$, which corresponds to $d(0)=0.02 \mathrm{~m}$, when moving backwards.

The third test consisted in a sinusoidal path (variable curva-
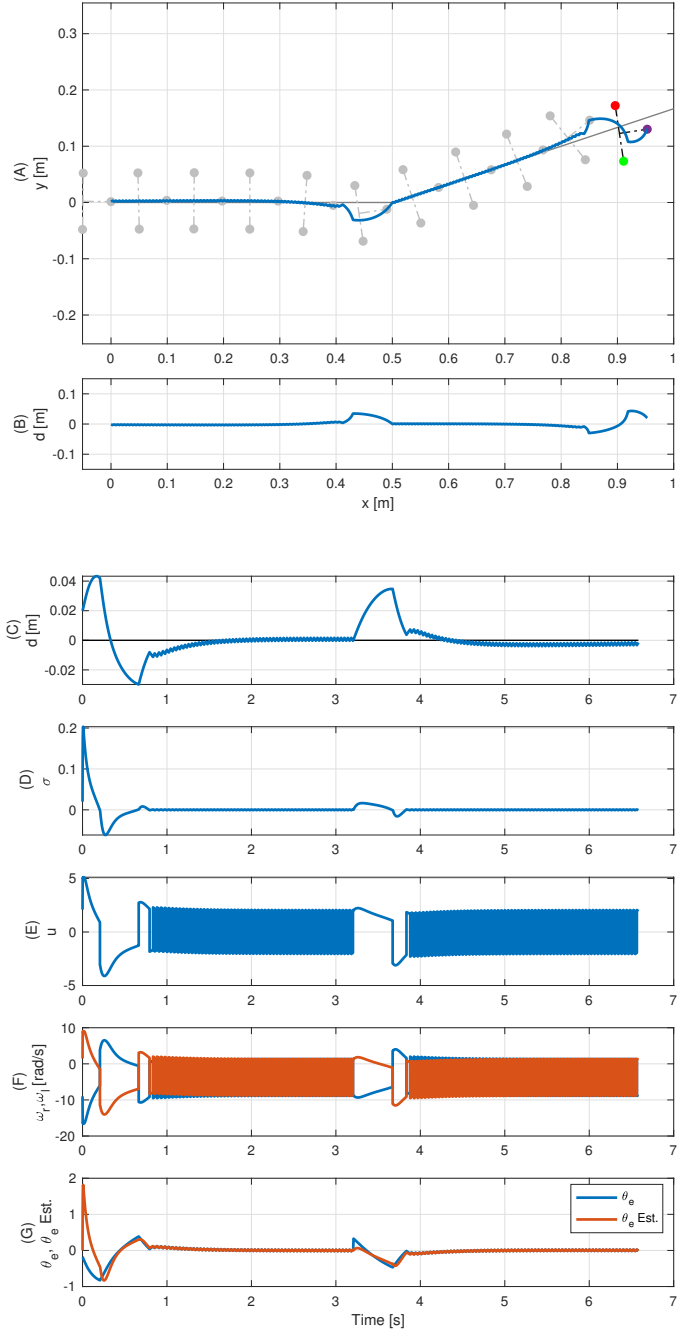

Fig. 4. Simulation results for two straight lines with an angle of $30^{\circ}$, moving backwards $\left(v_{x}<0\right)$ : (A) $x, y$ plane plot: distance sensor (blue dot), left wheel (red dot) and right wheel (green dot); (B) distance to the path, $d$, with respect to $x$; (C) distance to the path, $d$ (in blue), and its reference, $d^{\text {ref }}=0$ (in black); (D) sliding variable, $\sigma$; (E) control action, $u$; (F) wheel's speeds, $\omega_{l}, \omega_{r}$; and $(\mathrm{G})$ estimated deviation angle $\hat{\theta}_{e}$ (in red), with respect to the real one $\theta_{e}$ (in blue).

ture, $c \neq c$ ct.). The initial conditions were $x_{r}(0)=-0.0024 \mathrm{~m}$, $y_{r}(0)=-0.0123 \mathrm{~m}$ and $\theta(0)=0.15 \mathrm{rad}$, which corresponds to $d(0)=0.025 \mathrm{~m}$, when moving forwards, and $x_{r}(0)=$ $0.9036 \mathrm{~m}, y_{r}(0)=0.1228 \mathrm{~m}$ and $\theta(0)=0.15 \mathrm{rad}$, which corresponds to $d(0)=0.02 \mathrm{~m}$, when moving backwards.

Figures from 3 to 8 show the obtained results moving the differential robot both forward and backwards along the different paths. For the cases with constant curvature, see Figures from 3 to 6 , the robot is able to track the path and $d$ tends to zero. In addition, when moving forwards, the convergence to $d=0$ is fast because $\beta=0$ (even when the curvature is non-constant, see Figure 7). During backwards 

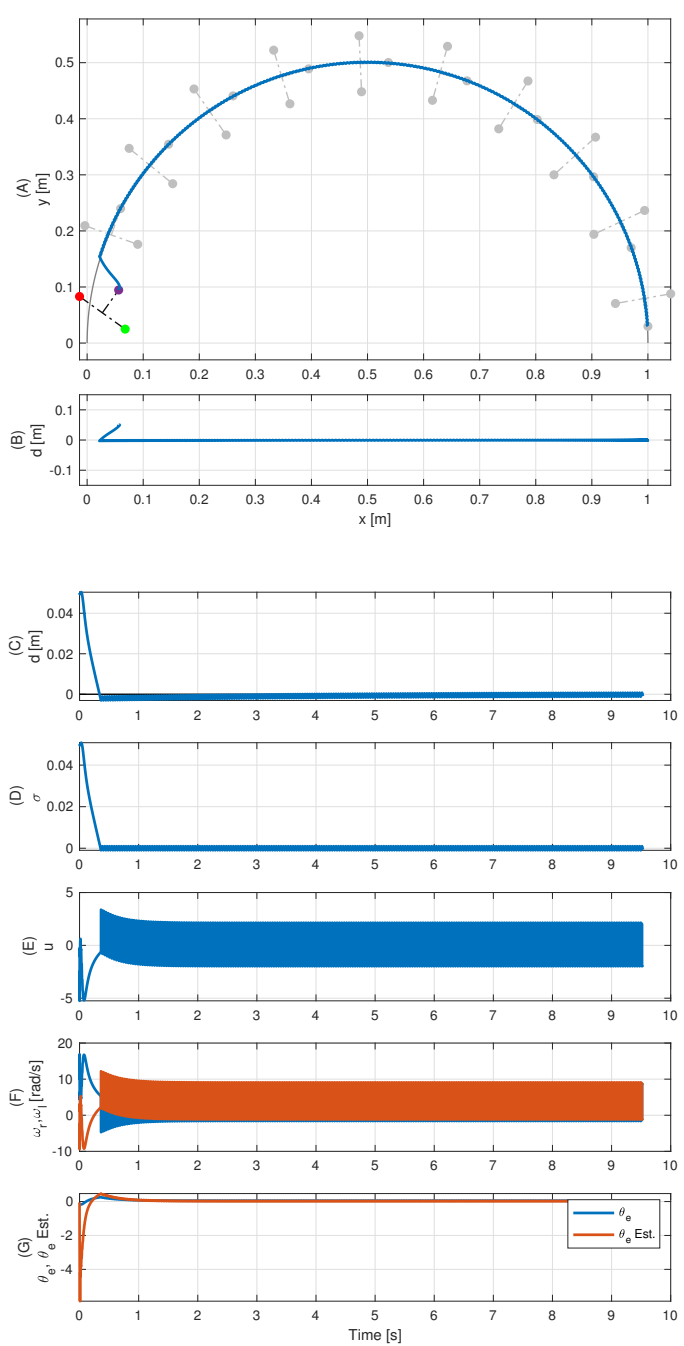

Fig. 5. Simulation results for a circular path with radius $0.5 \mathrm{~m}$, moving forwards $\left(v_{x}>0\right)$ : (A) $x, y$ plane plot: distance sensor (blue dot), left wheel (red dot) and right wheel (green dot) (B); distance to the path, $d$, with respect to $x$; (C) distance to the path, $d$ (in blue), and its reference, $d^{\text {ref }}=0$ (in black); (D) sliding variable, $\sigma$; (E) control action, $u$; (F) wheel's speeds, $\omega_{l}, \omega_{r}$; and (G) estimated deviation angle $\hat{\theta}_{e}$ (in red), with respect to the real one $\theta_{e}$ (in blue).

motions the performance decays because of the need of the state feedback $(\beta \neq 0)$, and the sliding motion is lost when the curvature in non-constant, see Figure 8. As expected, the switching control strategy results in a discontinuous control action and switching required speeds in the wheels. Finally, the proposed linear observer for the deviation angle presents a good performance and approximates the value of $\theta_{e}$, see (G) subplots in all the Figures.

\section{CONCLUSIONS}

This paper proposes a control strategy, based on sliding modes, for a differential-drive mobile robot. The main contribution of this paper, with respect to previous literature, is the possibility of moving both forward and backwards.
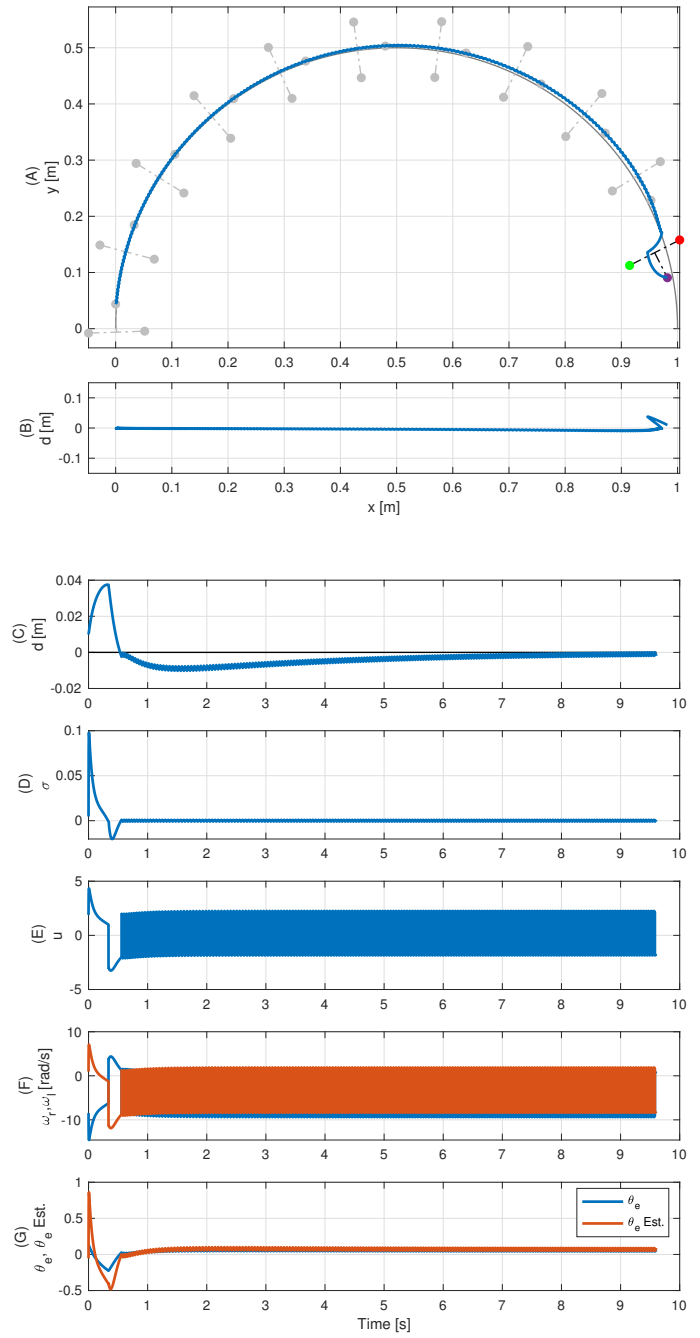

Fig. 6. Simulation results for a circular path with radius $0.5 \mathrm{~m}$, moving backwards $\left(v_{x}<0\right)$ : (A) $x, y$ plane plot: distance sensor (blue dot), left wheel (red dot) and right wheel (green dot) (B); distance to the path, $d$, with respect to $x$; (C) distance to the path, $d$ (in blue), and its reference, $d^{\text {ref }}=0$ (in black); (D) sliding variable, $\sigma$; (E) control action, $u$; (F) wheel's speeds, $\omega_{l}, \omega_{r}$; and (G) estimated deviation angle $\hat{\theta}_{e}$ (in red), with respect to the real one $\theta_{e}$ (in blue).

Future works include: i) considering a variable curvature (specially when moving backwards), ii) the stability analysis of the whole closed loop system (controller+observer), iii) the design of a higher order sliding mode controller to avoid the chattering effect observed in the simulations, and iv) the implementation in a real robot.

\section{REFERENCES}

[1] T.H. Lee, H.K. Lam, F.H.F. Leung, and P.K.S. Tam. A practical fuzzy logic controller for the path tracking of wheeled mobile robots. IEEE Control Systems Magazine, 23(2):60-65, 2003.

[2] H. Mehrjerdi, M. Saad, and J. Ghommam. Hierarchical fuzzy cooperative control and path following for a team of mobile robots. IEEE/ASME Trans. on Mechatronics, 16(5):907-917, 2011.

[3] Q. Zhang, L. Lapierre, and X. Xiang. Distributed control of coordinated path tracking for networked nonholonomic mobile vehicles. IEEE Trans. on Industrial Informatics, 9(1):472-484, 2013. 

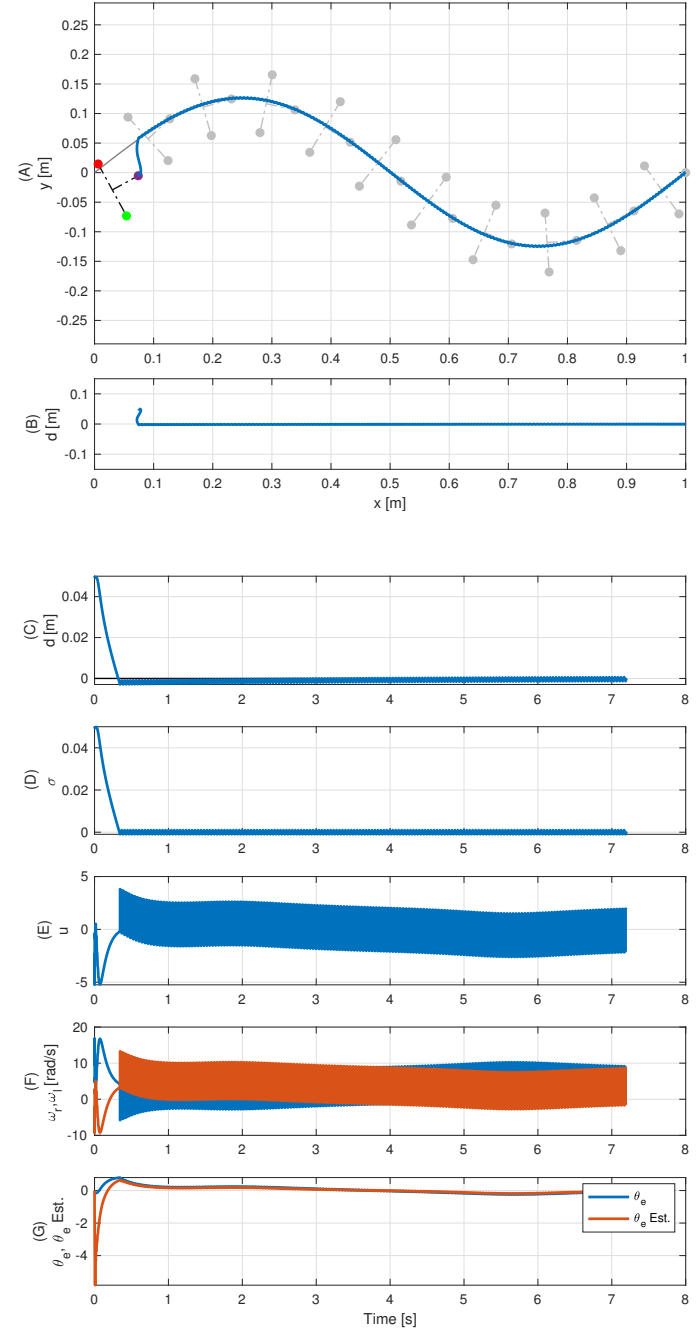

Fig. 7. Simulation results for a sinusoidal path, moving forwards $\left(v_{x}>0\right)$ : (A) $x, y$ plane plot: distance sensor (blue dot), left wheel (red dot) and right wheel (green dot); (B) distance to the path, $d$, with respect to $x$; (C) distance to the path, $d$ (in blue), and its reference, $d^{\text {ref }}=0$ (in black); (D) sliding variable, $\sigma$; (E) control action, $u$; (F) wheel's speeds, $\omega_{l}, \omega_{r}$; and (G) estimated deviation angle $\hat{\theta}_{e}$ (in red), with respect to the real one $\theta_{e}$ (in blue).

[4] T. Liu and Z-P. Jiang. Distributed formation control of nonholonomic mobile robots without global position measurements. Automatica, 49(2):592-600, 2013.

[5] G. Klancar, D. Matko, and S. Blazic. A control strategy for platoons of differential drive wheeled mobile robot. Robotics and Autonomous Systems, 59(2):57-64, 2011.

[6] G. Oriolo, A. De Luca, and M. Vendittelli. WMR control via dynamic feedback linearization: Design, implementation, and experimental validation. IEEE Trans. on Control Systems Technology, 10(6):835-852, 2002.

[7] B.M. Kim and P. Tsiotras. Controllers for unicycle-type wheeled robots: Theoretical results and experimental validation. IEEE Trans. on Robotics and Automation, 18(3):294-307, 2002.

[8] Z-P. Jiang and H. Nijmeijer. Tracking control of mobile robots: A case study in backstepping. Automatica, 33(7):1393-1399, 1997.

[9] S. Blazic. On periodic control laws for mobile robots. IEEE Trans. on Industrial Electronics, 61(7):3660-3670, 2014.

[10] J. Huang, C. Wen, W. Wang, and Z-P. Jiang. Adaptive output feedback tracking control of a nonholonomic mobile robot. Automatica,
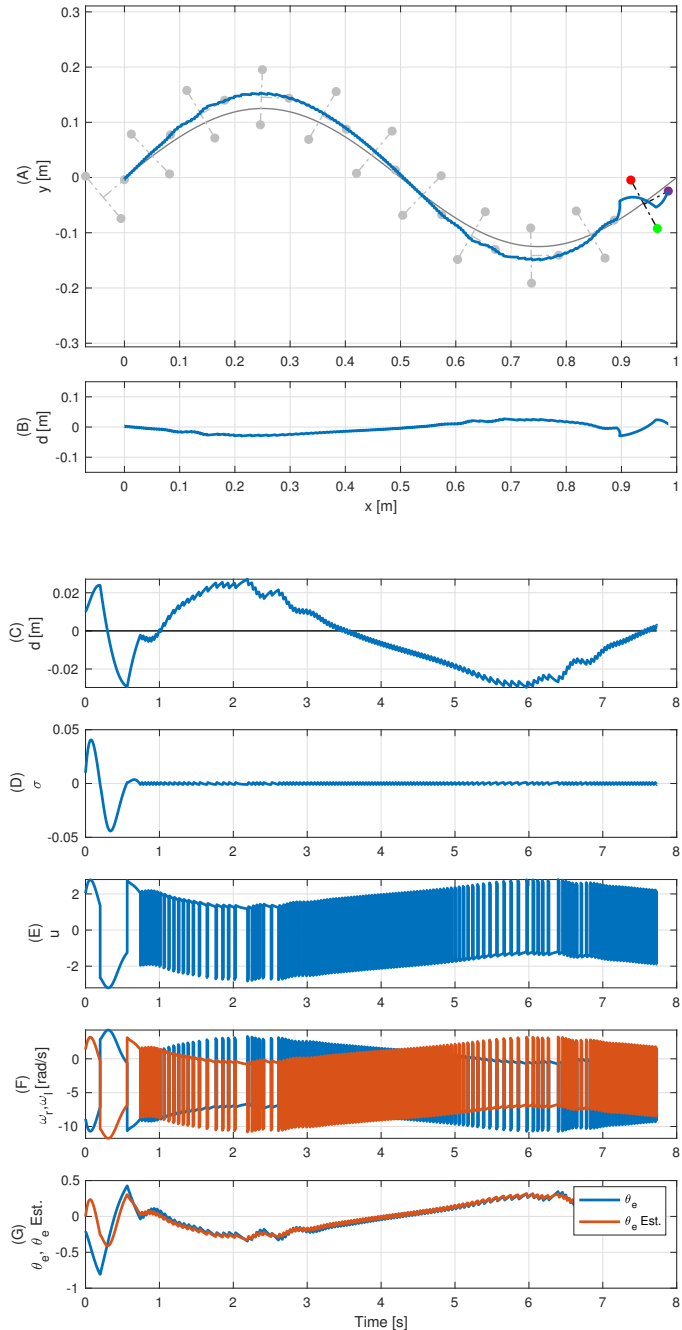

Fig. 8. Simulation results for a sinusoidal path, moving backwards $\left(v_{x}<0\right)$ : (A) $x, y$ plane plot: distance sensor (blue dot), left wheel (red dot) and right wheel (green dot); (B) distance to the path, $d$, with respect to $x$; $(\mathrm{C})$ distance to the path, $d$ (in blue), and its reference, $d^{\text {ref }}=0$ (in black); (D) sliding variable, $\sigma ;(\mathrm{E})$ control action, $u$; $(\mathrm{F})$ wheel's speeds, $\omega_{l}, \omega_{r}$; and (G) estimated deviation angle $\hat{\theta}_{e}$ (in red), with respect to the real one $\theta_{e}$ (in blue).

50(3):821-831, 2014

[11] J. Mu, X-G. Yan, S. K. Spurgeon, and Z. Mao. Nonlinear sliding mode control of a two-wheeled mobile robot system. Int. Journal of Modelling, Identification and Control, 27(2):75-83, 2017.

[12] D. Chwa. Sliding-mode tracking control of nonholonomic wheeled mobile robots in polar coordinates. IEEE Trans. on Control Systems Technology, 12(4):637-644, 2004.

[13] A. Ferrara and M. Rubagotti. Second-order sliding-mode control of a mobile robot based on a harmonic potential field. IET Control Theory Applications, 2(9):807-818, 2008.

[14] P. Morin and C. Samson. Motion control of wheeled mobile robots. In B. Siciliano and O. Khatib, editors, Handbooks of Robotics, chapter 34, pages 779-826. Springer, 2008.

[15] B. Paden, M. Cap, S. Z. Yong, D. Yershov, and E. Frazzoli. A survey of motion planning and control techniques for self-driving urban vehicles. IEEE Trans. on Intelligent Vehicles, 1(1):33-55, 2016. 